rupture owing to high pressure or damage to the septa the air passes through the lower-pressure alveolar sheaths to the mediastinum via the lung roots. Passage of air from the mediastinum to the neck or retroperitoneal space tends to decompress the mediastinum. The appearance of subcutaneous emphysema in the neck in respiratory disorders usually indicates the presence of a pneumomediastinum. In children, pneumomediastinum tends to occur in two broad age groups-the immediate postnatal period and later childhood. The incidence in later childhood in this department over a 12-year period has been approximately 1 in 1,000 admissions, the commonest cause being bronchial asthma. One case requiring surgical decompression has been reported (McNicholl, 1960).

The cause of air leakage in this boy cannot be definitely stated. There may have been inapparent infective damage to some alveoli. However, it seems reasonably certain that the cause of the increased alveolar pressure leading to the air leakage was the expiratory effort and grunting associated with the ketotic hyperventilation, since the amount and force of coughing observed were negligible.

A further point of interest was the delay in appearance of Hamman's sign until the air in the mediastinum had been almost absorbed, suggesting that this sign is most likely to be found when the pericardium and posterior surface of the sternum are not widely separated, allowing the systolic cardiac movement to "crunch" the air bubbles against the sternum. Consequently, the pressure of Hamman's sign may indicate a

\section{Pulmonary Angiography with External Cardiac Compression in Fatal Pulmonary Embolism}

\section{Brit. med. F., 1968, 4, 494-495}

In the following case of a young man who suddenly developed cardiac arrest after an acute massive pulmonary embolism a successful pulmonary angiogram was obtained while the external cardiac compression was continued to maintain an effective circulation.

\section{Case Report}

A man aged 38 was admitted to hospital on 14 November 1967 as a case of barbiturate poisoning. He was successfully treated by intermittent positive pressure ventilation and conventional medical therapy. On 29 November he suddenly collapsed with cardiac arrest. There had been no preliminary signs or symptoms to suggest an earlier pulmonary embolism, nor was there any clinical evidence of peripheral vein thrombosis. After external cardiac compression and other resuscitative measures the pupil size returned to normal, there was a spontaneous heart beat with a reasonable pulse volume at the wrist, and spontaneous though ineffective breathing was achieved. An electrocardiogram (lead III) showed a sinus tachycardia with right bundle-branch block and a few extrasystoles. Within three minutes, and without external cardiac compression, the circulation slowly failed, the pupils dilated, and the pulse disappeared, with cessation of cardiac beat and asystole on the electrocardiogram. The cardiac compression was therefore restarted, with good results. The same sequence was repeated every time the compression was stopped, and consequently the external cardiac compression was thereafter continuously employed along with other measures.

The presumptive diagnosis was confirmed by selective pulmonary arteriography. The pulmonary artery pressure was $35 \mathrm{~mm}$. $\mathrm{Hg}$ and systemic systolic pressure $50 \mathrm{~mm}$. An angiogram showed poor and slow filling of the main artery and particularly the left small rather than a large amount of air in the mediastinum. The extensive inspiratory crunching sounds along the anterior diaphragmatic attachment were most probably due to air between the parietal pleura and chest wall and in the loose areolar tissue along the pleurodiaphragmatic reflection.

We wish to thank Mr. D. Kneafsey for some useful discussion on intrathoracic anatomy and mechanics.

B. MCNICHOLL, M.D., M.R.C.P., D.C.H., Professor of Paediatrics.

J. P. MuRRay, M.D., F.F.R., D.M.R.D., Lecturer in Radiology.

B. EgAN, M.B., D.C.H., Paediatric Registrar.

P. McHuGH, M.B., Paediatric Intern.

University College and Regional Hospital, Galway.

\section{REFERENCES}

Bierman, C. W. (1967). Amer. F. Dis. Child., 114, 42.

Hamman, I. (1937). Trans. Ass. Amer. Physcns, 52, 311 Macklin, M. T., and Macklin, C. C. (1944). Medicine (Baltimore), 23, 281 .

McNicholl, B. (1960). Arch. Dis. Childh., 35, 389. pulmonary artery, which also had a block in its lower-lobe branch (see Fig.). The cardiac compression was constantly maintained except when the pulmonary and systemic arterial pressures were being recorded and the contrast medium was being injected.

The patient was then taken to the theatre and put on peripheral supportive bypass, the femoral vein and common femoral artery being used. This procedure immediately improved his circulatory state, the cardiac compression was no longer needed, and the systemic systolic arterial pressure registered $80 \mathrm{~mm}$. With the central and complete cardiopulmonary bypass the embolus was removed in many pieces, mainly from the left pulmonary arterial tree. For half an hour after the bypass the circulatory state remained satisfactory with a systemic pressure of $100 / 70 \mathrm{~mm} . \mathrm{Hg}$ and venous pressure of $10 \mathrm{~cm}$. of saline. Thereafter the cardiac performance deteriorated. While the left ventricle continued to contract vigorously, the right dilated and became flaccid, with rising venous pressure and low cardiac output. Finally the heart stopped. The unsuccessful outcome was due to delayed surgery, as it was

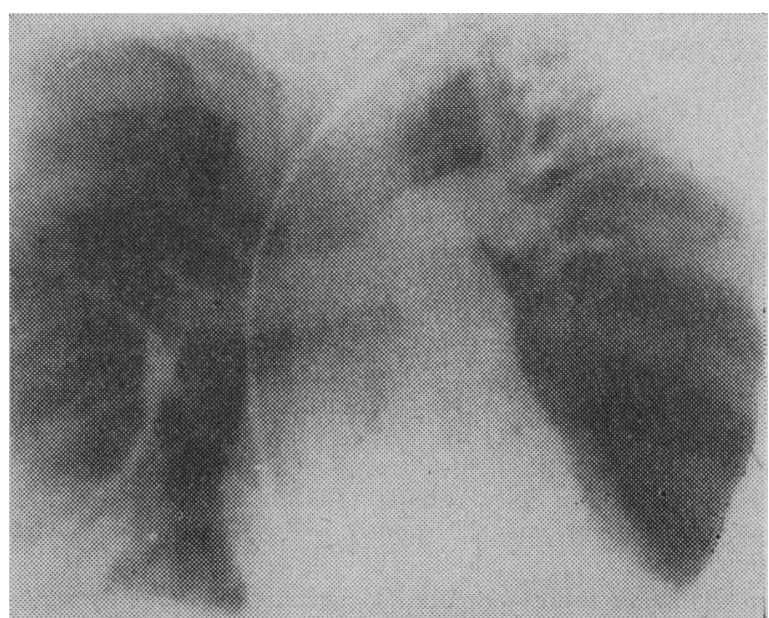

Angiogram showing block in lower-lobe branch of the left pulmonary artery. 
about two hours after the cardiac arrest that the pulmonary obstruction was bypassed. Post-mortem examination showed a tear in the left lobe of the liver and the haemoperitoneum, but no residual pulmonary emboli.

\section{COMMENT}

Massive pulmonary embolism often defies definitive clinical diagnosis. Cross and Mowlem (1967) reported a mortality of $100 \%$ in nine cases of erroneous diagnoses and attempted pulmonary embolectomies. Similar incidences were mentioned by Berger et al. (1968) and by Diacoff et al. (1968). The high mortality rate in pulmonary embolectomy has in part been related to errors in diagnosis, which can of course be prevented by the routine use of preoperative pulmonary angiography. Diacoff et al. (1968) reported on a few patients who underwent pulmonary angiography immediately after resuscitation from cardiac arrest ; none died as a result of this procedure, and in some who subsequently died necropsy did not show any evidence to suggest a deleterious effect from angiography in ill patients.

Pulmonary angiography is a safe and sure means of diagnosing a massive embolism. The above case, so far the first of its kind in the literature, shows that a diagnostic selective pulmonary angiogram of good quality can be obtained even when external cardiac compression is needed to support the circulation.

H. P. Gautam, M.S., F.R.C.S., Cardiothoracic Surgical Unit, Royal Infirmary, University of Manchester.

\section{REFERENCES}

Berger, R. L., Ryan, T. J., and Sidd, J. J. (1968). Surg. Clin. N. Amer.,

Cross, F. S., and Mowlem, A. (1967). Circulation, 35, Suppl. No. 1, p. 86.

Diacoff, G. R., Ranniger, K., and Moulder, P. V. (1968). Surg. Clin. N. Amer., 48, 71 .

\section{Long-term Electrode Catheter Pacing from Coronary Sinus}

Brit. med. F., 1968, 4, 495-496

Electrode catheter pacing from the coronary sinus is usually characterized by a rapid rise in threshold and early pacing failure (Siddons and Sowton, 1967). We report the case of a patient who was inadvertently, but successfully, paced from the coronary sinus for 16 months.

\section{CASE RePORT}

A 75-year-old woman was referred to the Royal Melbourne Hospital in July 1966 with a two-month history of recurrent chest pain. She had been found to have complete heart block and was being treated with ephedrine, but on the day of admission she had three Stokes-Adams attacks. On her admission the heart rate was 52 a minute, and an electrocardiogram showed complete heart block with multifocal ventricular extrasystoles. Serum enzyme studies confirmed recent infarction. An Elema EMT588 unipolar pacemaker catheter was passed to what was considered to be the apex of the right ventricle from the long saphenous vein in the groin and was connected to a fixed-rate external pacemaker unit, the indifferent electrode having been implanted in the abdominal wall. The patient returned to sinus rhythm with only intermittent paced beats, but when pacing was discontinued three weeks later the rate fell to 54 a minute with a 2 to 1 atrioventricular block, and so a permanent pacemaker generator (Devices) was implanted in the upper abdomen. Postoperatively a wound infection necessitated repositioning of the pacemaker unit, but regular pacing continued and the electrocardiogram showed a right bundle-branch block type of pattern (Fig. 1). In September 1967 the patient was admitted to hospital with a cerebrovascular accident characterized by deterioration in conscious state over two days, and hemiplegia, following which she recovered enough to be able to lead a wheel-chair existence.

The pacemaker generator was tested every three months (Knuckey et al., 1965) for a total of 16 months. The pacemaker readings remained unchanged until February 1968, when it was noted that the rate had risen from 74 to 79 beats a minute and the pulse width had decreased by $14 \%$, from 0.91 to 0.78 millisecond. The unit, however, continued to pace well with a one-to-one response. In accordance with our previous experience with Devices pacemakers, it was decided to replace the unit electively, but the patient was found dead in her bed at home before this could be carried out. Permission for only a limited necropsy was obtained.

The catheter in the groin, which ran subcutaneously to the abdominal unit, was in a satisfactory condition, as was the indifferent electrode. The heart weighed $480 \mathrm{~g}$. and the left ventricular myocardium was $1.5 \mathrm{~cm}$. in thickness. The coronary vessels showed moderate atherosclerosis, but there was no actual obstruction. There. was patchy fibrosis in the myocardium of the posterior part of the left ventricle. The pacemaker catheter was traced up the inferior vena

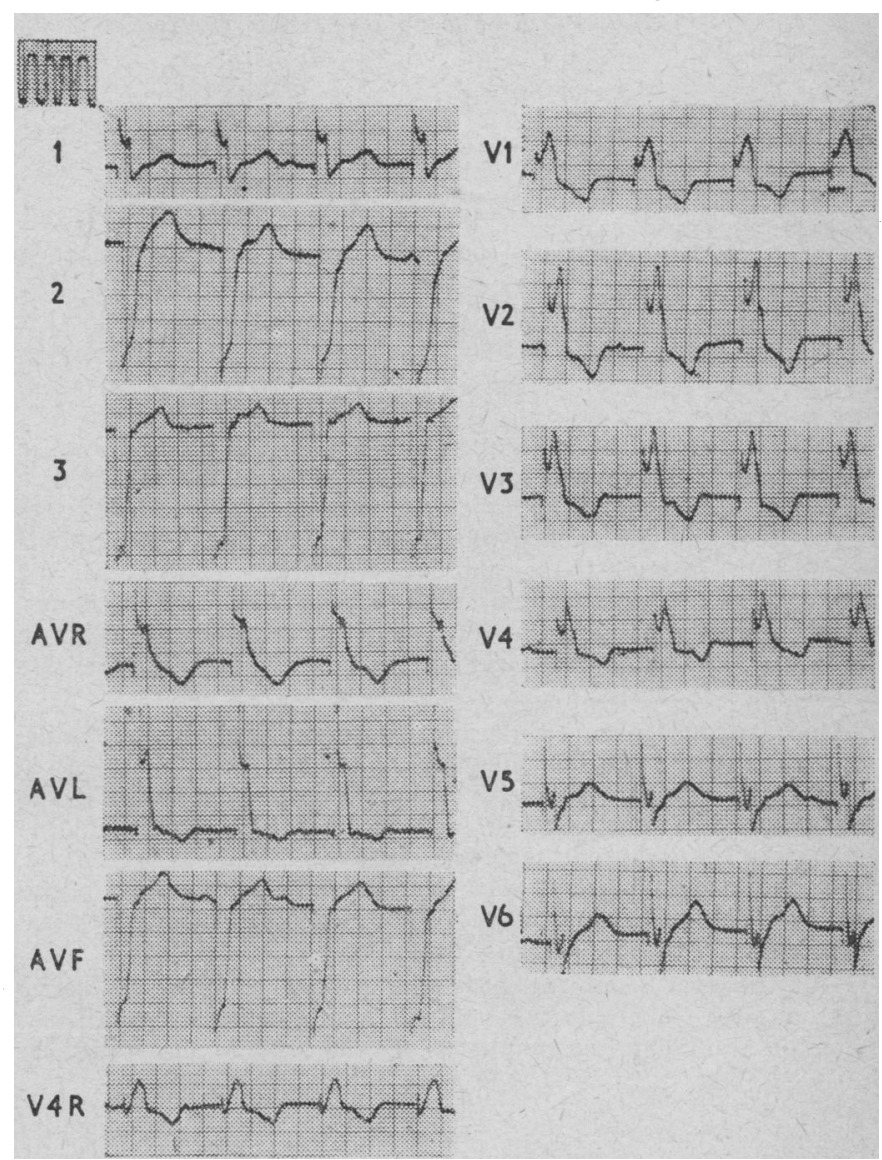

FIG. 1.-Electrocardiogram showing paced beats with a right bundlebranch block type of pattern. 